\title{
Si diffusion in GaAs
}

\author{
P MURUGAN, R POTHIRAJ ${ }^{\dagger}$, S D D ROY ${ }^{\ddagger}$ and K RAMACHANDRAN* \\ School of Physics, Madurai Kamaraj University, Madurai 625 021, India \\ ${ }^{\dagger}$ Department of Physics, Sri SRNM College, Sattur 626 203, India \\ Permanent address: NM Christian College, Martandam 629 165, India
}

MS received 2 April 2001

\begin{abstract}
Theoretical studies are carried out to ascertain the dominant mechanism of Si diffusion in GaAs. Lattice dynamical model calculations have shown that the most probable diffusion mechanism is through a single vacancy even though several experiments cannot fix the mechanism as substitutional, substitutionalinterstitial pair or neutral defect pair.
\end{abstract}

Keywords. Solid state diffusion; lattice dynamics; impurity diffusion.

\section{Introduction}

Impurity diffusion in semiconductors has the technological importance in the fabrication of junction devices, semi-insulating compounds and high temperature growth of materials (Mizoguchi et al 1999). The study on GaAs : Si diffusion has gained momentum in the last decade and over 25 papers have been published in various journals on the account of technological importance of the system (Fisher 1997). Persistent photoconductivity and the DX centres in this system are examples (Wolk et al 1991) for the interest in the system. Similarly, the LOPC (LO phonon-plasmon coupled) modes appear in such compounds (Dobal et al 1995). In addition, charge conversion takes place on $\mathrm{H}^{+}$ion implantation in this compound and this is manifested in the LOPC modes in Raman spectra (Murugan et al 2002). Si diffusion in GaAs has been experimentally studied by many techniques; among them, the measurements by secondary ion mass spectroscopy (SIMS) and capacitance-voltage method are important. In these methods, the diffusivity is obtained from the concentration profile and temperature.

Ahlgren et al (1997) have reported from their experimental studies on $\mathrm{Si}$ diffusion in GaAs, that concentration independent diffusion is mainly through interstitials, whereas concentration dependent diffusion is through vacancies. Greiner and Gibbons (1984) have also reported similar results from their experiments. Meanwhile, Lee et al (1990) reported that $\mathrm{Si}$ diffusion is through neutral defect pair, $\mathrm{Si}_{\mathrm{Ga}}^{+}-\mathrm{V}_{\mathrm{Ga}}^{-}$, which is also supported by many of the experiments (Nutt et al 1991; Matsushita et al 1993) and theoretical first-principle calculations (Dabrowski and Northrup 1994) and molecular dynamics simulations (Chen et al 1994).

\footnotetext{
*Author for correspondence
}

Herzog et al (1995) have reported, from SIMS, that Si diffusion in GaAs is through (i) interstitial and (ii) frank faulted loop and a kind of interstitial. Since different mechanisms for $\mathrm{Si}$ diffusion in GaAs are reported in literature and also no two diffusion experiments by the same technique could lead to identical results, we investigated by lattice dynamical approach the diffusion mechanism in this system, accounting for the experimental observations.

\section{Theory}

Diffusion of an atom in a crystal takes place through discrete jumps between the defect and the diffusing atom. In general, the role of these defects on diffusion also should be considered. Usually, only the passive role, considering the lattice relaxation to be negligible, due to the presence of defects when diffusion process takes place is considered to elucidate the diffusion process (Sadaiyandi and Ramachandran 1991). However, the active role taking into account the lattice relaxation for the defects is considered in our present approach (Sadaiyandi and Ramachandran 1992). When this jump is obstructed by a ring of neighbouring atoms, the diffusing atom should overcome the potential barrier for the jump to materialize. Instantaneous relative displacement of the diffusing atom with respect to the neighbours in the diffusion ring is expressed as reaction coordinate (Flynn 1968)

$$
\mathbf{X}=\left[\mathbf{U}^{\alpha}-\frac{1}{n} \sum_{i} \mathbf{U}^{i}\right] \cdot \hat{\mathbf{X}},
$$

where $\mathbf{U}^{\alpha}$ is the displacement of the diffusing atom, $\mathbf{U}^{i}$ the displacement of the $i$ th atom in the ring of $n$ neighbours obstructing the jump, and $\hat{\mathbf{X}}$, the unit vector along the jump direction. 
The displacements of atoms due to the presence of impurity is considered as a perturbed term through the scattering matrix. When a lattice plane wave, $\mathbf{U}_{0}$, passes through the defect space consisting of the defect and the neighbours in the diffusion ring, the situation can be treated as a scattering problem with $\mathbf{U}$ as the displacement matrix consisting the displacement of the scattered wave w, following the theory of Maradudin et al (1971)

$$
\mathbf{U}=\mathbf{U}_{0}+\mathbf{w},
$$

where the matrix, $\mathbf{U}_{0}$, corresponds to a perfect lattice and can be expressed as

$$
\mathbf{U}_{0}(\mathbf{q}, k)=\left[\frac{\hbar}{2 N M_{k} \omega(\mathbf{q}, k)}\right]^{1 / 2} \mathbf{e}(\mathbf{q}, k) \exp (i \mathbf{q} \cdot \mathbf{r})
$$

and

$$
\mathbf{L} \mathbf{U}_{0}=0 \text {, }
$$

where $\mathbf{e}(\mathbf{q}, k)$ is the polarization vector in the wave vector q for $k$ th atom in the $l$ th cell. $M_{k}$ is the mass of the $k$ th atom. The matrix, $L$, is constructed from the force constants, $\phi$, as

$$
L_{\alpha \beta}\left(l l^{\prime} ; k k^{\prime} ; \omega^{2}\right)=M_{k} \omega^{2} \delta_{l l^{\prime}} \delta_{k k^{\prime}} \delta_{\alpha \beta}-\phi_{\alpha \beta}^{0}\left(l l^{\prime} ; k k^{\prime}\right),
$$

$\alpha$ and $\beta$ are the degrees of freedom. $\mathbf{w}$ the scattered wave matrix is defined as

$$
\mathbf{w}=\mathbf{G}\left(\frac{\partial \mathbf{L}}{\mathbf{I}-\mathbf{G} \partial \mathbf{L}}\right) \mathbf{U}_{0} .
$$

The displacements of the host atoms in the defect space are given in the form of a column vector $\mathbf{U}$, as

$$
\mathbf{U}=\left(\mathbf{I}+\frac{\mathbf{G} \partial \mathbf{L}}{\mathbf{I}-\mathbf{G} \partial \mathbf{L}}\right) \mathbf{U}_{0}
$$

where I is the unit matrix, G the Green's functions matrix, $\mathbf{L}$ corresponds to the vibrations of the perfect host crystal and $\partial \mathbf{L}$ the perturbation matrix, all matrices being of the order $3(n+1) \times 3(n+1)$.

The atomic jump rate then can be written as (Achar 1970)

$$
\Gamma=\Gamma_{0} \exp \left[\frac{-\mathbf{X}_{\mathbf{c}}^{2}}{\sum_{q, k}|\mathbf{X}(\mathbf{q}, k)|^{2}}\right],
$$

where

$$
\Gamma_{0}=\sqrt{\frac{\sum_{q, k} \omega^{2}(\mathbf{q}, k)|\mathbf{X}(\mathbf{q}, k)|^{2}}{\sum_{q, k}|\mathbf{X}(\mathbf{q}, k)|^{2}}},
$$

and $\Gamma_{0}$ is called attempt frequency of the jump. $\mathbf{X}_{\mathrm{c}}$ is the critical value of $\mathbf{X}$ above which only, the jump will materialize. Critical value of $\mathbf{X}_{\mathrm{c}}$ is obtained from

$$
\frac{\mathbf{X}_{c}^{2}}{\sum_{q, k}|\mathbf{X}(\mathbf{q}, k)|^{2}}=\frac{Q}{k_{\mathrm{B}} T},
$$

where $Q$ is the activation energy, $k_{\mathrm{B}}$ the Boltzmann constant and $T$ the temperature.

Using the jump frequency, the diffusion constant, $D_{0}$, and diffusion coefficient, $D$, can be estimated from

$$
\begin{aligned}
& D_{0}=\frac{H \Gamma_{0} d^{2}}{2 \pi s}, \\
& D=D_{0} \exp \left(\frac{-Q}{k_{\mathrm{B}} T}\right),
\end{aligned}
$$

where $H$ is the Havan's ratio, $d$ the jump distance and $s$ the number of identical jumps. $Q$ is the activation energy for jump. For the vacancy mechanism, $H$ and $s$ are 0.78146 and 12, respectively, as GaAs belongs to zinc blende structure (Sadaiyandi 1992).

\section{Diffusion mechanism}

Three kinds of diffusion mechanisms are considered in our present work viz. single vacancy mechanism, vacancy assisted interstitial and neutral defect pair (divacancy) mechanism, since many of the experiments suggest these possible mechanisms.

\subsection{Single vacancy mechanism}

In this process the substitutional foreign- or host-atom diffuses by jumping into the nearby vacancy, which acts as the diffusion vehicle. Particularly, in the case of Si diffusion in GaAs, $\mathrm{Si}$ is initially assumed to occupy a positively charged substitutional site, $\mathrm{Si}_{\mathrm{Ga}}^{+}$at $\left(\begin{array}{lll}0 & 0 & 0\end{array}\right)$. It diffuses by jumping into nearby negatively charged vacant site $\mathrm{V}_{\mathrm{Ga}}^{-}$at $\left(\begin{array}{lll}1 & 1 & 0\end{array}\right)$. The ring of neighbours obstructing the jump is shown in figure 1 and has only three atoms in the ring at the positions $\mathrm{Ga}\left(\begin{array}{lll}0 & 1 & 1\end{array}\right), \mathrm{Ga}$ $\left(\begin{array}{lll}0 & \overline{1} & 1\end{array}\right)$ and As $\left(\frac{1}{2} \frac{1}{2} \frac{1}{2}\right)$, lying in a plane perpendicular to the jump direction [ $\left[\begin{array}{lll}1 & 1 & 0\end{array}\right]$.

\subsection{Vacancy assisted interstitial}

Since, it is reported that $\mathrm{Si}$ diffusion in GaAs by vacancy assisted interstitial mechanism may be possible, it is also considered in the present work and the mode of transport by this mechanism is shown in figure 2 . The jumping $\mathrm{Si}$ atom is initially assumed to be at $\left(\begin{array}{lll}0 & 0 & 0\end{array}\right)$ site; first goes to the interstitial site at $\left(\frac{1}{2} \frac{1}{2} \frac{\overline{1}}{2}\right)$ in [11 $\left.\overline{1}\right]$ direction and then 
to $\mathrm{Ga}$ vacancy at $\left(\begin{array}{lll}1 & 1 & 0\end{array}\right)$ site in $\left[\begin{array}{lll}1 & 1 & 1\end{array}\right]$ direction. The resulting jump direction is same as $\left[\begin{array}{lll}1 & 1 & 0\end{array}\right]$. The ring of neighbours obstructing these jumps is shown in figure 2 .

\subsection{Neutral defect pair}

In this mechanism, substitutional foreign- or self-atom diffuses by jumping into one vacancy of the bound defect pair. The $\mathrm{Si}$ atom is assumed to occupy a $\mathrm{Ga}$ site first substitutionally. Because of the fluctuations due to lattice vibration, the $\mathrm{Si}$ atom jumps to the As site $\left(\frac{3}{2} \frac{1}{2} \frac{1}{2}\right)$ which is purely a temporary site and the $\mathrm{Si}$ atom will occupy this intermediate metastable site for diffusion. Immediately it jumps to the next vacant Ga (2 111$)$ site. Two vacancies are assumed, one at Ga site and other at As site, so that $\mathrm{Si}$ diffuses through these sites as these are the two possible vacancies through which the jump can occur. The system may be unstable for the two defects to occur in the same unit cell simultaneously, but the As vacancy is assumed only as an intermediate site and the present configuration of $\left[\mathrm{V}_{\mathrm{Ga}}-\mathrm{V}_{\mathrm{As}}\right]$ is postulated. The direction of first jump is [ [ $\left.\begin{array}{lll}1 & 1 & 1\end{array}\right]$ and for the second jump

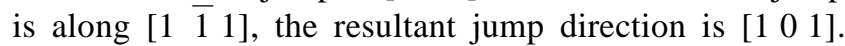
Figure 3 illustrates this mechanism of diffusion.

\section{Isotope effect}

Achar's (1970) theory gives the method of finding the most probable diffusion mechanism from isotope effect $(\Delta K)$, which is defined as the ratio of kinetic energy of the moving atom, in the dissolution normal mode at the

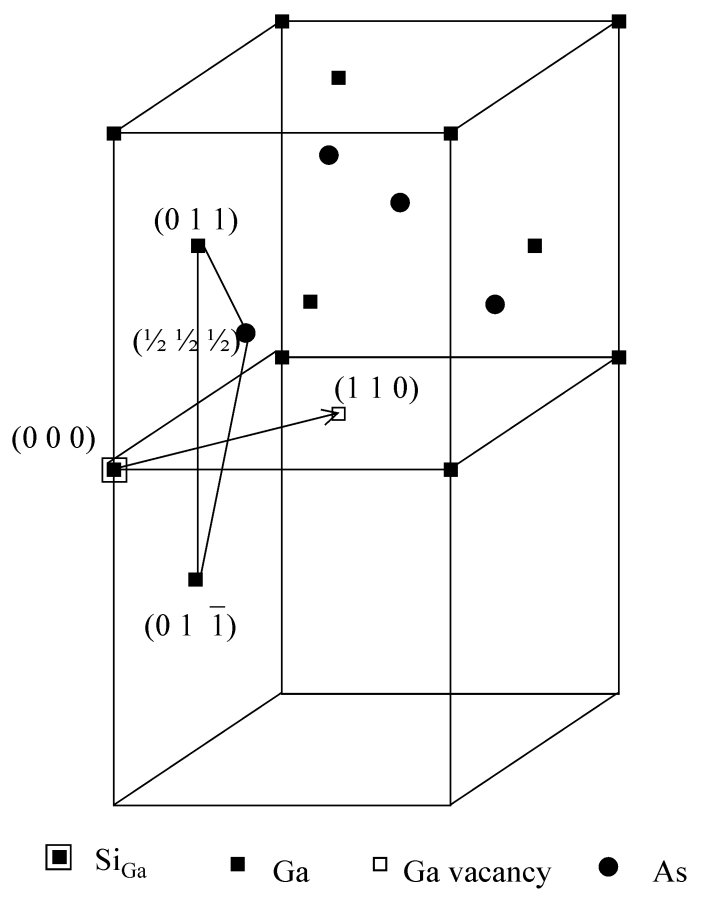

Figure 1. Single vacancy mechanism (positions are given in units of $r_{0}=a / 2$ ). saddle point, to the total kinetic energy. The kinetic energy and the isotope effect are correlated by a factor, $f$, through the relation

$$
\Delta E=f \Delta K \text {. }
$$

The isotope effect is a measure of the dependence of the tracer diffusion rate on the isotope mass, and is given by

$$
\Delta K=\frac{\frac{\Gamma_{1}}{\Gamma_{2}}-1}{\sqrt{\frac{M_{2}}{M_{1}}}-1} .
$$

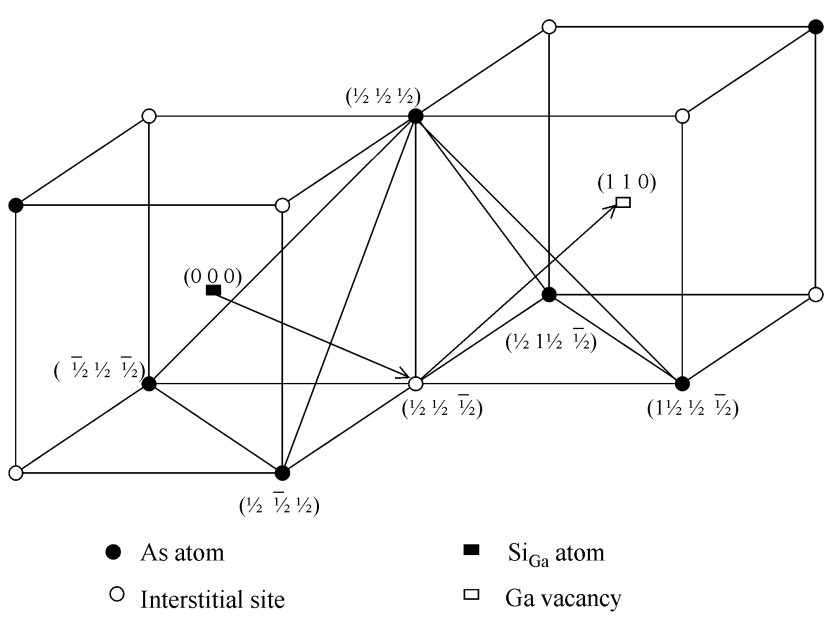

Figure 2. Vacancy assisted interstitial (positions are given in units of $r_{0}=a / 2$ ).

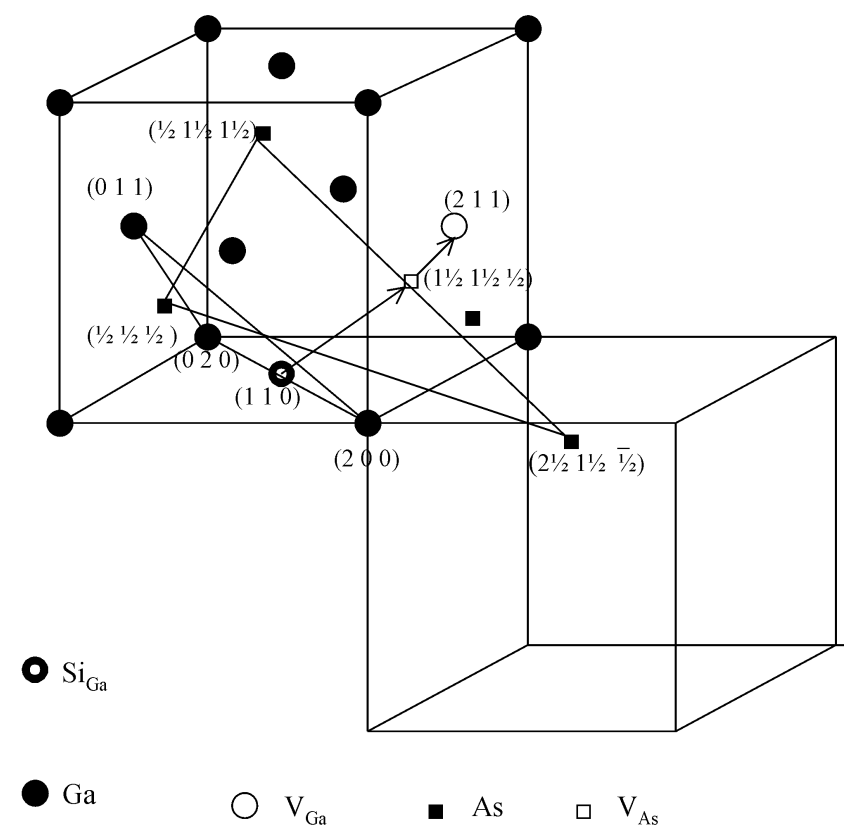

Figure 3. Neutral defect pair mechanism (position of atoms are given in units of $a / 2$ ). 
Here $M_{1}$ and $M_{2}$ are the masses of the two most abundant isotopes of diffusing atoms. Jump frequencies of isotopes are computed from the reaction coordinate and the mechanism yielding the isotope effect exceeding $50 \%$ is generally considered as the most probable.

\section{Results}

The jump frequency and diffusion coefficient for different temperatures are computed by employing the scattering matrix theory for the three different mechanisms viz. single vacancy, vacancy assisted interstitial and neutral defect pair mechanism.

In all the above cases, most abundant isotopes of $\mathrm{Si}$, namely ${ }^{28} \mathrm{Si}$ and ${ }^{29} \mathrm{Si}$ are considered for diffusion. Green's

Table 1. The jump frequencies and isotope effect for Si diffusion in GaAs (Active role - single vacancy mechanism).

\begin{tabular}{lcccc}
\hline $\begin{array}{l}\text { Temp. } \\
(\mathrm{K})\end{array}$ & $\begin{array}{c}\text { Isotope } \\
(\mathrm{Si})\end{array}$ & $\begin{array}{c}X_{\mathrm{c}} \\
(\%)\end{array}$ & $\begin{array}{c}\Gamma \\
(\mathrm{rad} / \mathrm{sec})\end{array}$ & $\begin{array}{c}\Delta K \\
(\%)\end{array}$ \\
\hline \multirow{2}{*}{700} & 28 & 1.358 & $0.4655 \times 10^{-4}$ & 87.3 \\
& 29 & 1.339 & $0.4728 \times 10^{-4}$ & \\
900 & 28 & 1.357 & 0.3861 & 87.5 \\
& 29 & 1.338 & 0.3922 & \\
1100 & 28 & 1.356 & $0.1204 \times 10^{3}$ & 87.3 \\
& 29 & 1.336 & $0.1229 \times 10^{3}$ & \\
1300 & 28 & 1.356 & $0.6414 \times 10^{4}$ & 87.7 \\
& 29 & 1.336 & $0.6515 \times 10^{4}$ & \\
\hline
\end{tabular}

$X_{\mathrm{c}}$ values are given in $\%$ of atomic radius of cation atom $(\mathrm{Ga})$. functions are obtained from phonon frequencies and eigen vectors of $\mathrm{GaAs}$ for entire Brillouin zone from the modified rigid ion model (Plumelle and Vandevyver 1976). For constructing the scattering matrix, the lattice relaxation is considered through the force constant change (Pothiraj and Ramachandran 1995). Reaction coordinate of diffusing atom is calculated from (1), considering all the equivalent symmetry points. Initially, attempt frequencies are estimated for various diffusion processes. From the attempt frequency, $X_{\mathrm{c}}$, the critical values of $X$ and diffusion constant are estimated. The jump frequency is estimated from (8) and the isotope effect is evaluated from the jump frequencies for all diffusion mechanisms at various temperatures from (14). Necessary software have been developed and the jump frequency and diffusion constant are computed.

The $X_{\mathrm{c}}$, critical value of reaction coordinate, jump frequencies and the isotope effect for single vacancy mechanism are reported in table 1 . It is found that the isotope effect, $\Delta K$, at all temperatures is well above $87 \%$ and the mean value of $X_{\mathrm{c}}$ is around $1.345 \%$ of the ionic radius of $\mathrm{Ga}^{3+}$.

In the vacancy assisted interstitial mechanism, two jumps are separately considered, one jump to interstitial site and another to vacant site. Jump frequency is estimated from the geometric mean of the two jump frequencies. Isotope effect (table 2) demonstrates that the $\Delta K$ is around $58 \%$ and the mean $X_{\mathrm{c}}$ around $1.69 \%$ of ionic radius of $\mathrm{Ga}^{3+}$.

In the case of neutral defect pair mechanism, two jumps are involved similar to the above case, viz. first jump to vacant As site and then subsequently to a vacant

Table 2. The jump frequencies and isotope effect for Si diffusion in GaAs (Active role - vacancy assisted interstitial).

\begin{tabular}{|c|c|c|c|c|c|c|}
\hline $\begin{array}{l}\text { Temp. } \\
(\mathrm{K})\end{array}$ & $\begin{array}{l}\text { Isotope } \\
\text { (Si) }\end{array}$ & Jumps & $\begin{array}{c}X_{\mathrm{c}} \\
(\%)\end{array}$ & $\begin{array}{l}\text { Jump frequencies } \\
(\mathrm{rad} / \mathrm{sec})\end{array}$ & $\begin{array}{c}\Gamma \\
(\mathrm{rad} / \mathrm{sec})\end{array}$ & $\begin{array}{l}\Delta K \\
(\%)\end{array}$ \\
\hline \multirow{2}{*}{700} & 28 & $\begin{array}{l}\text { I } \\
\text { II }\end{array}$ & $\begin{array}{l}2 \cdot 019 \\
1 \cdot 478\end{array}$ & $\begin{array}{l}0.3761 \times 10^{-4} \\
0.5014 \times 10^{-4}\end{array}$ & $0.4343 \times 10^{-4}$ & \multirow{2}{*}{$59 \cdot 7$} \\
\hline & 29 & $\begin{array}{l}\text { I } \\
\text { II }\end{array}$ & $\begin{array}{l}1.926 \\
1.421\end{array}$ & $\begin{array}{l}0.3787 \times 10^{-4} \\
0.5087 \times 10^{-4}\end{array}$ & $0.4389 \times 10^{-4}$ & \\
\hline \multirow{2}{*}{900} & 28 & $\begin{array}{l}\text { I } \\
\text { II }\end{array}$ & $\begin{array}{l}2 \cdot 018 \\
1 \cdot 477\end{array}$ & $\begin{array}{l}0.3094 \\
0.4128\end{array}$ & $0 \cdot 3574$ & \multirow{2}{*}{$59 \cdot 1$} \\
\hline & 29 & $\begin{array}{l}\text { I } \\
\text { II }\end{array}$ & $\begin{array}{l}1.925 \\
1.420\end{array}$ & $\begin{array}{l}0.3115 \\
0.4188\end{array}$ & $0 \cdot 3612$ & \\
\hline \multirow{2}{*}{1100} & 28 & $\begin{array}{l}\text { I } \\
\text { II }\end{array}$ & $\begin{array}{l}2 \cdot 017 \\
1 \cdot 476\end{array}$ & $\begin{array}{l}0.9597 \times 10^{2} \\
1.2811 \times 10^{2}\end{array}$ & $1 \cdot 1088 \times 10^{2}$ & \multirow{2}{*}{58.9} \\
\hline & 29 & $\begin{array}{c}\text { I } \\
\text { II }\end{array}$ & $\begin{array}{l}1 \cdot 925 \\
1 \cdot 419\end{array}$ & $\begin{array}{l}0.9662 \times 10^{2} \\
1.2995 \times 10^{2}\end{array}$ & $1 \cdot 1205 \times 10^{2}$ & \\
\hline \multirow{2}{*}{1300} & 28 & $\begin{array}{l}\text { I } \\
\text { II }\end{array}$ & $\begin{array}{l}2 \cdot 017 \\
1 \cdot 476\end{array}$ & $\begin{array}{l}0.5095 \times 10^{4} \\
0.6804 \times 10^{4}\end{array}$ & $0.5889 \times 10^{4}$ & \multirow{2}{*}{$58 \cdot 7$} \\
\hline & 29 & $\begin{array}{l}\text { I } \\
\text { II }\end{array}$ & $\begin{array}{l}1.924 \\
1.419\end{array}$ & $\begin{array}{l}0.5130 \times 10^{4} \\
0.6901 \times 10^{4}\end{array}$ & $0.5950 \times 10^{4}$ & \\
\hline
\end{tabular}


Ga site. Jump frequency and isotope effect are computed (table 3) and it is found that the isotope effect for this case is about $52 \%$ and the $X_{c}$ is around $1.63 \%$ of ionic radius of $\mathrm{Ga}^{3+}$.

\section{Conclusions}

Even though extensive experimental results are available for $\mathrm{Si}$ diffusion in $\mathrm{GaAs}$, there is no clear-cut conclusion on the mechanism of diffusion i.e. the single vacancy and neutral defect pair are some of the possibilities reported. In order to understand the exact mode of Si diffusion in GaAs, we have investigated theoretically all the possible mechanisms.

The values of $\Delta K$, a measure of the kinetic energy necessary for overcoming the potential barrier so that diffusion is possible, for the single vacancy mechanism are about $87 \%$ for all temperatures from $700-1300 \mathrm{~K}$, whereas in vacancy assisted interstitial process, $\Delta K$ values are only about $59 \%$. By comparison of $\Delta K$ values obtained with the two prominent mechanisms, single

Table 3. The jump frequencies and isotope effect for Si diffusion in GaAs (Active role neutral defect pair).

\begin{tabular}{|c|c|c|c|c|c|c|}
\hline $\begin{array}{l}\text { Temp. } \\
\text { (K) }\end{array}$ & $\begin{array}{l}\text { Isotope } \\
\quad(\mathrm{Si})\end{array}$ & Jumps & $\begin{array}{c}X_{\mathrm{c}} \\
(\%)\end{array}$ & $\begin{array}{c}\text { Jump frequencies } \\
(\mathrm{rad} / \mathrm{sec})\end{array}$ & $\begin{array}{c}\Gamma \\
(\mathrm{rad} / \mathrm{sec})\end{array}$ & $\begin{array}{l}\Delta K \\
(\%)\end{array}$ \\
\hline \multirow[b]{2}{*}{700} & 28 & $\begin{array}{l}\text { I } \\
\text { II }\end{array}$ & $\begin{array}{l}1.8077 \\
1.5529\end{array}$ & $\begin{array}{l}0.4653 \times 10^{-4} \\
0.4947 \times 10^{-4}\end{array}$ & $0.4798 \times 10^{-4}$ & \multirow{2}{*}{$52 \cdot 5$} \\
\hline & 29 & $\begin{array}{l}\text { I } \\
\text { II }\end{array}$ & $\begin{array}{l}1.7350 \\
1.4825\end{array}$ & $\begin{array}{l}0.4699 \times 10^{-4} \\
0.4991 \times 10^{-4}\end{array}$ & $0.4843 \times 10^{-4}$ & \\
\hline \multirow{2}{*}{900} & 28 & $\begin{array}{l}\text { I } \\
\text { II }\end{array}$ & $\begin{array}{l}1 \cdot 8064 \\
1.5516\end{array}$ & $\begin{array}{l}0 \cdot 3832 \\
0 \cdot 4071\end{array}$ & $0 \cdot 3950$ & \multirow{2}{*}{$52 \cdot 4$} \\
\hline & 29 & $\begin{array}{l}\text { I } \\
\text { II }\end{array}$ & $\begin{array}{l}1.7337 \\
1.4812\end{array}$ & $\begin{array}{l}0.3869 \\
0 \cdot 4108\end{array}$ & $0 \cdot 3987$ & \\
\hline \multirow{2}{*}{1100} & 28 & $\begin{array}{l}\text { I } \\
\text { II }\end{array}$ & $\begin{array}{l}1.8057 \\
1.5510\end{array}$ & $\begin{array}{l}1.1892 \times 10^{2} \\
1.2632 \times 10^{2}\end{array}$ & $1 \cdot 2256 \times 10^{2}$ & \multirow{2}{*}{$52 \cdot 5$} \\
\hline & 29 & $\begin{array}{l}\text { I } \\
\text { II }\end{array}$ & $\begin{array}{l}1.7330 \\
1.4806\end{array}$ & $\begin{array}{l}1.2008 \times 10^{2} \\
1.2746 \times 10^{2}\end{array}$ & $1 \cdot 2371 \times 10^{2}$ & \\
\hline \multirow{2}{*}{1300} & 28 & $\begin{array}{l}\text { I } \\
\text { II }\end{array}$ & $\begin{array}{l}1 \cdot 8053 \\
1.5506\end{array}$ & $\begin{array}{l}0.6316 \times 10^{4} \\
0.6708 \times 10^{4}\end{array}$ & $0.6509 \times 10^{4}$ & \multirow{2}{*}{$52 \cdot 5$} \\
\hline & 29 & $\begin{array}{l}\text { I } \\
\text { II }\end{array}$ & $\begin{array}{l}1.7327 \\
1.4802\end{array}$ & $\begin{array}{l}0.6377 \times 10^{4} \\
0.6768 \times 10^{4}\end{array}$ & $0.6570 \times 10^{4}$ & \\
\hline
\end{tabular}

Table 4. Diffusivity, $D\left(\mathrm{~cm}^{2} / \mathrm{sec}\right)$ of ${ }^{28} \mathrm{Si}$ in GaAs for various mechanisms with experimental results.

\begin{tabular}{|c|c|c|c|c|}
\hline \multirow{2}{*}{$\begin{array}{l}\text { Temperature } \\
(\mathrm{K})\end{array}$} & \multicolumn{3}{|c|}{$D$ (Present calculation) } & \multirow[b]{2}{*}{ Experiment } \\
\hline & I & II & III & \\
\hline 700 & $0.799 \times 10^{-19}$ & $0.721 \times 10^{-19}$ & $0.797 \times 10^{-19}$ & $\begin{array}{l}\mathbf{0 . 5 2 1} \times \mathbf{1 0}^{-\mathbf{1 9}}(\mathrm{a}) \\
0.222 \times 10^{-21}(\mathrm{~b})\end{array}$ \\
\hline 900 & $0.658 \times 10^{-15}$ & $0.593 \times 10^{-15}$ & $0.656 \times 10^{-15}$ & $\begin{array}{c}\mathbf{0 . 2 9 3} \times \mathbf{1 0}^{-\mathbf{1 6}}(\mathrm{a}) \\
0.537 \times 10^{-17}(\mathrm{~b})\end{array}$ \\
\hline 1100 & $0 \cdot 204 \times 10^{-12}$ & $0 \cdot 184 \times 10^{-12}$ & $0 \cdot 203 \times 10^{-12}$ & $\begin{array}{l}\mathbf{0 . 1 6 5} \times \mathbf{1 0}^{-14}(\mathrm{a}) \\
0.327 \times 10^{-14}(\mathrm{~b})\end{array}$ \\
\hline 1300 & $0 \cdot 108 \times 10^{-10}$ & $0.978 \times 10^{-11}$ & $0 \cdot 108 \times 10^{-10}$ & $\begin{array}{l}0.268 \times 10^{-13}(a) \\
0.278 \times 10^{-12}(b)\end{array}$ \\
\hline
\end{tabular}

I. Single vacancy mechanism;

II. Vacancy assisted interstitial, resultant diffusivity has been taken from the geometric mean of two jumps diffusivity;

III. Neutral defect pair mechanism, resultant diffusivity has been taken from the geometric mean of two jumps diffusivity;

(a) Laikhtman et al 1991; (b) Hirai et al 1995;

Bold values are close to our results. 
vacancy is found to be the most dominant mechanism. Since neutral defect pair mechanism is also reported from experiments, we have worked out this mechanism also theoretically and the $\Delta K$ values are found to be about $52 \%$.

In conclusion, we have calculated the isotope effect for (i) single vacancy, (ii) single vacancy assisted interstitial and (iii) neutral defect pair mechanisms to be $87 \%, 59 \%$, $52 \%$, respectively for active role of the defects. These results indicate that the most dominant mechanism for $\mathrm{Si}$ diffusion in GaAs is through single vacancy compared to the other two mechanisms under normal condition. The other mechanisms may also be possible under specific conditions, either at elevated pressure or temperature or higher doping concentrations.

In fact, most possible mechanism can also be understood from $X_{\mathrm{c}}$, the critical value of reaction coordinate $X$. In case of single vacancy mechanism, this has a lower value compared to the other mechanisms, as shown in tables $1-3$.

The results of the diffusivity for these mechanisms are given in table 4, where we could find a reasonable agreement with the experimental values (Laikhtman et al 1991; Hirai et al 1995). Diffusivity of $\mathrm{Si}$ in GaAs has nearly identical values for both single vacancy and neutral defect pair mechanisms and it is lesser in vacancy assisted interstitials mechanism.

\section{Acknowledgements}

The authors acknowledge Prof. N Krishnamurthy for the fruitful discussions they had with him and one of the authors (PM) acknowledges the financial support from the Department of Science and Technology, New Delhi, in the form of a fellowship.

\section{References}

Achar B N N 1970 Phys. Rev. B2 3848

Ahlgren T, Likonen J, Slotte J, Raisanen J, Rajatora M and Keinonen J 1997 Phys. Rev. B56 4597
Chen B, Zhang W M and Bernholc J 1994 Phys. Rev. B49 2985

Dabrowski J and Northrup J E 1994 Phys. Rev. B49 14286

Dobal P S, Bist H D, Mehta S K and Jain R K 1995 J. Appl. Phys. 773934

Fisher D J 1997 Diffusion in GaAs and other III-V semiconductors: 10 Years of research (Switzerland: Scitec Publications)

Flynn C P 1968 Phys. Rev. 171682

Greiner M E and Gibbons J F 1984 Appl. Phys. Lett. 44750

Herzog L, Egger U, Breitenstein O and Hettwer H G 1995 Mater. Sci. \& Eng. B30 43

Hirai M, Ohnishi H, Fujita K, Vaccaro P and Watanabe T 1995 J. Cryst. Growth 150209

Laikhtman B, Kiehl R A and Frank D J 1991 J. Appl. Phys. 70 1531

Lee J L, Wei L, Tanigawa S and Kawabe M 1990 J. Appl. Phys. 685571

Nutt H C, Smith R S, Towers M, Rees P K and James D J 1991 J. Appl. Phys. 70821

Maradudin A A, Montroll E W, Weiss G H and Ipatova I P 1971 Theory of lattice dynamics in the harmonic approximation (New York: Academic Press) Suppl. 3

Matsushita S, Terada S, Fujii E and Harada Y 1993 Appl. Phys. Lett. 63225

Mizoguchi K, Miyamaru F, Nakajima M, Hase M and Nakashima S 1999 Physica B272 367

Murugan P, Kesavamoorthy R, Amirthapandian S, Saravanan R, Ramachandran K and Krishnamurthy N 2002 Physica B315 56

Plumelle P and Vandevyver M 1976 Phys. Status Solidi (b) 73 271

Pothiraj R and Ramachandran K 1995 Phys. Status Solidi (b) 18771

Sadaiyandi K 1992 Lattice dynamical investigations on some semiconducting crystals, Ph.D. thesis, Madurai Kamaraj University, Madurai

Sadaiyandi K and Ramachandran K 1991 Phys. Status Solidi (b) $\mathbf{1 6 5} 327$

Sadaiyandi K and Ramachandran K 1992 Phys. Status Solidi (b) 170 K77

Wolk J A, Kruger M B, Heyman J N, Walukiewicz W, Jeanloz R and Haller E E 1991 Phys. Rev. Lett. 66774 